\title{
Theory of De-Pinning of Monolayer Films Adsorbed on a Quartz Crystal Microbalance
}

\author{
J. B. Sokoloff* and I. Webman**+ \\ *Physics Department and Center for Interdisciplinary Research on Complex Systems, \\ Northeastern University, Boston, MA 02115; ** Physics Department, \\ Bar-Ilan University, Ramat-Gan, Israel, ${ }^{+}$Deceased.
}

(Dated: October 24, 2018)

\begin{abstract}
In quartz crystal microbalance (QCM) studies of the friction between an adsorbed monolayer film and a metallic substrate, the films are observed to slide relative to the substrate under inertial forces of order $10^{-14} d y n$ per film atom, a force much smaller than all theoretical estimates of the force that surface defects are capable of exerting. In this letter we propose, in order to resolve this issue, that if the defect potentials have a range of greater than an atomic spacing, the net force on a relatively stiff film due to the defects is likely to be extremely small. Line defects (e.g., step and facet edges and grain boundaries) as well as more localized defects (e.g., vacancies) are considered.
\end{abstract}

PACS numbers: 68.35.Af,62.20.-x

Quartz crystal microbalance (QCM) studies of monolayers of molecules on metallic substrates[1] provide detailed information about friction at the atomic level. The QCM consists of a quartz oscillator of frequency $\sim 10^{6} \mathrm{~Hz}$. Monolayer films adsorbed on the metallic surface of the quartz crystal oscillator are found to slip with respect to the surface during a period of the oscillator. Small changes observed in the frequency and in the associated Q factor allow us to gauge the friction between mono-layer and substrate. The amount of dissipation generated under most conditions implies that sliding motion of more than a lattice constant occurs during a period of the QCM.

For film atom mass $m \approx 10^{-22} \mathrm{~g}$, appropriate for xenon atoms, QCM frequency $\omega \approx 10^{7} \mathrm{rad} / \mathrm{s}$ and amplitude $A \approx 100 A^{\circ}$, which are appropriate parameters for the QCM experiment, the inertial force $m \omega^{2} A$ is only about $10^{-14}$ dyn per film atom. Calculations of pinning of a monolayer film by defects, based on both perturbation theory[2] and on molecular dynamic simulations[3] imply that this inertial force per atom due to oscillations of the substrate should not be sufficiently strong to overcome the pinning resulting from the defects. This is in fact what was found in experimental studies by Taborek[4] to attempt to reproduce the striking temperature dependence of the sliding friction found by Dayo and Krim for a nitrogen film sliding on a lead substrate, as the temperature dropped below the superconducting transition temperature of the lead[5]. It has been established [6], however, that the substrates used in this experiment were much more contaminated than those used in Ref. 5. In the usual treatment of an elastic medium interacting with a disordered potential [7], the range of the defect potential is assumed to be sufficiently small compared to a film lattice spacing, so that it can only interact with one film atom at a time. In contrast, pinning defects on the substrate typically extend over one or more atomic lengths. For example, the potential produced by line defects (e.g., step and facet edges and grain boundaries) clearly extend a number of lattice spacings along the length of the de- fect, and even a more localized defect such as a vacancy extends at least out past its nearest neighbor atoms, because the neighboring atoms will displace towards the missing atom, resulting in a potential which extends out at least that far. It will be argued here that for potentials, typical of surface defects, that have a long enough range to interact with two or more atoms along the sliding direction, the forces on these atoms tend to cancel each other, reducing the force on the film due to a single defect to a value much smaller than the maximum force that a defect can exert on a single film atom.

It has been established using simulations $[3$ that the Larkin length [7], which is a measure of the stiffness of the film, is many lattice spacings long implying that the film distorts over distances long compared to the mean defect potential spacing, and hence certainly long compared to the defect potential width. It will now be demonstrated that because of the film stiffness, even for defect potentials with a range of a little more than a film lattice sp acing, the forces exerted on the film atoms tend to cancel to a great degree. (Incidentally, the simulations reported in Ref. 3 used a defect potential of much shorter range.) Consider the potential energy of a rigid film of atoms interacting with a single defect potential

$$
\sum_{\mathbf{R}} v(\mathbf{R}+\Delta \mathbf{r})
$$

where $v(\mathbf{R}+\Delta \mathbf{r})$ is the potential energy of an atom located at the point $\mathbf{R}+\Delta \mathbf{r}$ in the film due to the defect, $\Delta \mathbf{r}$ represents a displacement of the film relative to the potential and $\mathbf{R}$ is an atomic position in the periodic lattice of the film. Since this quantity is a periodic function of $\Delta \mathbf{r}$ with the periodicity of the film lattice, and hence, it can be expressed as a Fourier series [ 8$]$ in the coordinates in the plane parallel to the interface

$$
\sum_{\mathbf{R}} v(\mathbf{R}+\Delta \mathbf{r})=\sum_{\mathbf{G}} \bar{v}(\mathbf{G}, z) e^{i \mathbf{G} \cdot \Delta \mathbf{r}}
$$

where the Fourier coefficient at a distance $\mathrm{z}$ from the surface, $\bar{v}(\mathbf{G}, z)=\Omega^{-1} \int d^{2} r e^{-i \mathbf{G} \cdot \mathbf{r}} v(\mathbf{r})$, where $\Omega$ is the unit 
cell area and $\mathbf{G}$ is a reciprocal lattice vector of the film. The integral is taken over the whole film. For line defects, such as step and facet edges and grain boundaries $\mathrm{v}(\mathbf{r})$ will vary quite slowly as a function of $\mathbf{r}$ along the length of the line defect (defined as the distance over which the defect is relatively straight). As a consequence, we shall see that the Fourier coefficient $\bar{v}(\mathbf{G}, z)$ are likely to be quite small for general directions of $\mathbf{G}$, such that $\mathbf{G}$ has a component along the length of the defect sufficiently large compared with $2 \pi$ divided by the length of the defect, a condition which is easy to satisfy for any line defect long compared to a film lattice constant. The Fourier coefficients will also be very small for defects such as vacancies which are localized around a point on the substrate.

Since the exact form of the defect potential is not known, let us illustrate this effect by studying a couple of simple smooth potentials which drop off reasonably rapidly at large distances, in order to develop a picture of what one expects for defect potentials with a range greater than a lattice spacing. Here we will consider primarily a two dimensional Gaussian and Lorentzian potential, which represent two extremes, as the Gaussian falls to zero very rapidly at large distances while the Lorentzian falls off relatively slowly (as is evidenced by the fact that its second moment is infinite). For an anisotropic Gaussian defect potential, $v(\mathbf{r})=-V_{0}(z) e^{-\left[\left(x / b_{1}\right)^{2}+\left(y / b_{2}\right)^{2}\right]}$, where $b_{1}$ and $b_{2}$ are the range parameters, we obtain, for a triangular lattice $\bar{v}(\mathbf{G})=-2 V_{0} \pi\left(b_{1} b_{2} /(3)^{1 / 2} a^{2}\right) e^{-\left(G_{x}^{2} b_{1}^{2} / 4+G_{y}^{2} b_{2}^{2} / 4\right)}$ For the circularly symmetric two dimensional Lorentian potential $v(\mathbf{r})=-V_{0}(z)\left[1+\left(x / b_{1}\right)^{2}+\left(y / b_{2}\right)^{2}\right]^{-1}$, $\bar{v}(\mathbf{G}, z)$ on the right hand side of Eq. (2) is equal to $-\left[4 \pi b_{1} b_{2} / a^{2}(3)^{1 / 2}\right] V_{0} K_{0}(Q)$, where $Q=\left(G_{x}^{2} b_{1}^{2}+\right.$ $\left.G_{y}^{2} b_{2}^{2}\right)^{1 / 2}$ and $K_{0}(Q)$ is the spherical Hankel function of the first kind with an imaginary argument [9], whose large argument assymptotic form is $K_{0}(Q) \approx$ $(2 / \pi Q)^{1 / 2} e^{-Q}$. These forms can be used to model a line defect which runs along the $\mathrm{x}$-axis if we take $b_{1}>>b_{2}$, with $b_{1}$ representing the distance along the defect length that one must travel in order to reach a point at which the relatively straight section of the defect ends, and $b_{2}$ represents the width of the defect potential perpendicular to the direction of the line defect. We will assume the film's crystallographic axes and sliding direction to be at an arbitrary angle with respect to the $\mathrm{x}$ and $\mathrm{y}$ axes. If we wish to model localized defects, such as vacancies or interstitials, we set $b_{1}=b_{2}$. The magnitude of the Fourier coefficient is a very sensitive function of the range of the potential. By taking the negative of the gradient of Eq. (2) with respect to $\Delta \mathbf{r}$, we obtain the force on the film, whose maximum value is approximately $G \bar{v}(\mathbf{G}, z)$, where $\mathbf{G}$ is one of the smallest reciprocal lattice vectors. The maximum force on the film for the Gaussian defect potential for $b_{1}=b_{2}=b=1.23 a$ is $0.979 \times 10^{-7} V_{0} / b$ and for $b_{1}=b_{2}=b=0.5 a$ it is $0.0248 V_{0} / b$. The corresponding value for the maximum force for the two dimensional Lorentzian is $0.00349 V_{0} / b$ for $b_{1}=b_{2}=b=1.23 a$ and $0.0729 V_{0} / b$ for $b_{1}=b_{2}=b=0.5 a$. These quantities should be compared to the maximum possible force that these model potentials can exert on a single film atom, which is $0.429 V_{0} / b$ for the Gaussian and $0.5 V_{0} / b$ for the Lorentzian. We see that for both of these values of $b$ the maximum force on the film is much smaller than the maximum force that it can exert on a single atom for both model potentials and it is a very sensitive function of $b / a$.

For line defects, the effects can be even more dramatic. For example, for a line defect along the $\mathrm{x}$-axis, it is reasonable to assume that $b_{1}$ is a number of lattice spacings long, and hence, considerably longer than a film lattice spacing. Hence, for virtually all orientations of the film axes, $\left|G_{x} b_{1}\right|>>1$, and hence, we can see for the two dimensional Gaussian and Lorentzian potentials discussed in the last paragraph, their Fourier coefficients will be extremely shall, and hence we see from Eq. (2), the dependence of the interaction of the potential with the film with the defect on $\Delta \mathbf{r}$ will be extremely small, implying extremely small forces on the film.

Other model defect potentials give similar results. For example, the potentials $-V_{0} /\left[\cosh \left(x / b_{1}\right) \cosh \left(y / b_{2}\right)\right]$ and $-V_{0} /\left[\left(1+\left(x / b_{1}\right)^{2 n}\left(1+\left(y / b_{2}\right)^{2 n}\right]\right.\right.$, where $\mathrm{n}$ is any integer, also result in Fourier coefficients which fall off as exponential functions for reasonably large values of $\left|G_{x}\right| b_{1}$ and $\left|G_{y}\right| b_{2}$. The latter potential with a sufficiently large value of $\mathrm{n}$ is a good model for a line defect whose potential is nearly constant along most of its length and only has significant variation near its ends. The only requirement in order to get a Fourier coefficients which fall off rapidly at large values of its argument (i.e., exponentially or better) is that the potential due to the defect be smooth.

At any temperature, there will be lattice vibrations. At a given instant of time, a lattice vibration of wavevector $\mathbf{q}$ will add a term $\mathbf{A} \cos (\mathbf{q} \cdot(\mathbf{r}+\mathbf{R})-\omega t)$ to $\mathbf{R}$ in Eqs. (1), where $\mathbf{A}$ is the amplitude (assumed to be much smaller than a lattice constant a) and $\omega$ is the frequency of the vibrational mode and $\mathrm{t}$ is the time. Then, expanding $\mathrm{v}$ in a Fourier series, Eq. (1) becomes

$$
\sum_{\mathbf{r}} N^{-1} \sum_{\mathbf{k}} \bar{v}(\mathbf{k}, z) e^{i \mathbf{k} \cdot(\mathbf{R}+\Delta \mathbf{r})} e^{i \mathbf{k} \cdot \mathbf{A} \cos (\mathbf{q} \cdot(\mathbf{r}+\mathbf{R})-\omega t)},
$$

where $\mathrm{N}$ is the number of atoms in the film. Expanding the second exponential in Bessel functions Eq. (3) becomes

$$
N^{-1} \sum_{\mathbf{R}} \sum_{\mathbf{k}} \bar{v}(\mathbf{k}, z) \sum_{n=0}^{\infty} i^{n} J_{n}(\mathbf{k} \cdot \mathbf{A}) e^{i(\mathbf{k}-n \mathbf{q}) \cdot(\mathbf{R}+\Delta \mathbf{r})} e^{i n \omega t},
$$

which when summed over $\mathbf{R}$ becomes

$$
\sum_{\mathbf{G}} \sum_{n} i^{n} J_{n}((\mathbf{G}-n \mathbf{q}) \cdot \mathbf{A}) \bar{v}(\mathbf{G}-n \mathbf{q}, z) e^{i(\mathbf{G} \cdot \Delta \mathbf{r}-n \omega t)} .
$$

For the $\mathrm{n}=1$ term, which should be a good approximation for the small values of $|(\mathbf{G}-n \mathbf{q}) \cdot \mathbf{A}|$ characteristic of lattice vibrations, we find that $\bar{v}(\mathbf{G}-\mathbf{q}, z)$ for the Gaussian potential now contains a term $e^{-|\mathbf{G}-\mathbf{q}|^{2} b^{2} / 2}$, which can be 
considerably larger than $e^{-G^{2} b^{2} / 2}$, which occurs for $\mathrm{n}=0$, by a sufficient amount to make the former term dominate over the latter for values of $\mathrm{q}$ which are comparable in magnitude to the smallest values of $G$, even though it is multiplied by $J_{n}((\mathbf{G}-\mathbf{q}) \cdot \mathbf{A})$, which is much less than 1 . As the former term is proportional to $\mathbf{A}$ which oscillates in time for a lattice vibration, this term produces an oscillating term in the force on the film, which can be larger in magnitude than the force on the film that would occur if there were no vibrations [i.e., the $n=0$ term in Eq. (5)]. Thus at nonzero temperatures, the lattice vibrations can produce oscillating forces acting on the part of the film in the range of the potential, which dominate over the static pinning force on the film due to the potential under consideration, implying that at nonzero temperatures, the film will not be pinned by the defects because the film is pushed out of its total potential minimum by this force.

In the previous paragraphs, the film was taken to be completely rigid, and the distortion of the film resulting from its interaction with the defect potential was neglected. In order to examine the validity of this approximation, let us now consider the small distortion of the film resulting from the potential, in order to determine whether it will destroy the reduction of the potential's interaction with the film discussed in the previous two paragraphs. The displacement of a film atom located at point $\mathbf{R}$ is given in lowest order perturbation theory by

$$
\mathbf{u}(\mathbf{R}) \approx-\sum_{\mathbf{R}^{\prime}} \mathbf{G}\left(\mathbf{R}-\mathbf{R}^{\prime}\right) \cdot \nabla^{\prime} v\left(\mathbf{R}^{\prime}, z\right)
$$

where $\mathbf{G}\left(\mathbf{R}-\mathbf{R}^{\prime}\right)$ is the elasticity Green's function [8]. It is shown in Ref. [8] that

$$
\mathbf{G}\left(\mathbf{R}-\mathbf{R}^{\prime}\right)=N^{-1} \sum_{\mathbf{k}, \alpha} \frac{\hat{\epsilon}_{\mathbf{k}, \alpha} \hat{\epsilon}_{\mathbf{k}, \alpha} e^{i \mathbf{k} \cdot\left(\mathbf{R}-\mathbf{R}^{\prime}\right)}}{\omega_{\alpha}^{2}(\mathbf{k})}
$$

where $\omega_{\alpha}(\mathbf{k})$ is the frequency of the $\alpha^{t h}$ vibrational mode of wavevector $\mathbf{k}$ and $\hat{\epsilon}_{\mathbf{k}, \alpha}$ is the unit vector which gives its polarization. Substituting Eq. (6) in Eq. (6), we obtain

$$
\mathbf{u}(\mathbf{R}) \approx-N^{-1} i \sum_{\mathbf{k}, \alpha} \frac{\hat{\epsilon}_{\mathbf{k}, \alpha} \hat{\epsilon}_{\mathbf{k}, \alpha} e^{i \mathbf{k} \cdot \mathbf{R}}}{\omega_{\alpha}^{2}(\mathbf{k})} \cdot \mathbf{k} \bar{v}(\mathbf{k}, z) .
$$

If we add $\mathbf{u}$ to $\mathbf{R}$ in Eq. (1), linearize in $\mathbf{u}$ and substitute for $\mathbf{u}$ from Eq. (9), we find that the first order term in an expansion in powers of $\mathbf{u}$ for the force on the film is given by

$$
\left.N^{-1} \sum_{\mathbf{k}, \mathbf{G}} \frac{\left[(\mathbf{G}-\mathbf{k}) \cdot \hat{\epsilon}_{\mathbf{k}, \alpha}\right]\left[\mathbf{k} \cdot \hat{\epsilon}_{\mathbf{k}, \alpha}\right]}{\omega_{\alpha}^{2}(\mathbf{k}}\right) \bar{v}(\mathbf{G}-\mathbf{k}) \bar{v}(\mathbf{k}) e^{i \mathbf{k} \cdot \Delta \mathbf{r}} .
$$

We see from Eq. (9) that since we found earlier that $\bar{v}(\mathbf{k}, z)$ becomes negligibly small rapidly once $\mathrm{k}$ becomes larger than $1 / \mathrm{b}$, only Fourier components of wavevector larger than $1 / \mathrm{b}$ will make a significant contribution to Eq. (9). We shall now illustrate that the modulation of the film resulting from the defect potential should have

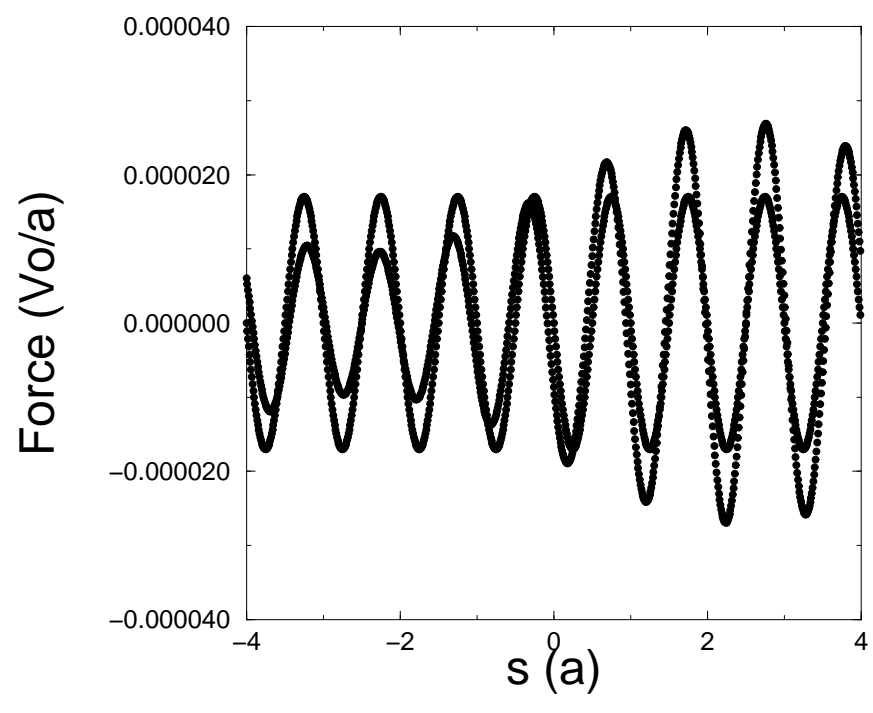

FIG. 1: The total force due to a Gaussian potential acting on a rigid lattice of atoms of spacing a is plotted as a function of the displacement of the film $\mathrm{s}$ in the lower amplitude curve for $b=1.236067978 \mathrm{a}$. The total force acting on a modulated lattice is shown in the higher amplitude curve.

little effect on our conclusion that the defect interacts extremely weakly with the adsorbed film with a simple calculation. Consider a stiff two dimensional square lattice of atoms of lattice constant a interacting with a Gaussian potential $-V_{0} e^{-r^{2} / b^{2}}$, were $\mathrm{r}$ is the distance from the center of the potential. We choose the range parameter of the potential, $b=1.23 \mathrm{a}$. For this choice of parameters the potential range is about one and a half lattice spacings. The lattice is slid a distance $\mathrm{s}$ and the total force due to this potential acting on it is plotted as a function of $\mathrm{s}$ (the lower amplitude curve in Fig. 1). The position of an atom in the lattice is then displaced by an amount $0.01 a \cos \left((2 \pi / \lambda) x_{n}\right)$ in the $\mathrm{x}$-direction, where the position of a film atom is $\left(x_{n}, y_{m}\right)=(n a, m a)$ where $\mathrm{n}$ and $\mathrm{m}$ are integers, to simulate the effect of this static modulation of the film. There is no displacement $\Delta x$ included because a modulation produced by the potential does not slide relative to the potential. Here, $\lambda$ is chosen to be $4 \mathrm{~b}$ (in the range of the shortest $\lambda$ value which makes a significant contribution in Eq. (10)), so that half of a wavelength extends across the width of the potential well. The results are given in Fig. 1. As can be seen the force on the film when such a modulation is present is comparable to that in the absense of a modulation, and is much less than the maximum force that a defect can exert on a film atom.

We have considered the interaction of a single defect with the film and have demonstrated that this can easily be much weaker than the maximum possible interaction of that defect with a single film atom. When we consider the interaction of the film with the random distribution of defects over the substrate that is typically found, we must consider the fact that in the weak pinning regime 
(in which the film is not able to distort enough to minimize its interaction with all of the randomly distributed defects), the forces due to the defects do not act in phase 7]. As a consequence, the net force per atom on the film gets reduced in addition by a factor of the square root of the number of defects within a Larkin domain, which is proportional to the ratio of the Larkin length and a film lattice constant. Since for a two dimensional solid the Larkin length is equal to the ratio of the elastic constant and the interaction with a single defect 11], the over-all interaction of the defects with the film is proportional to the square of the defect interaction, rather than being a linear function of it. Furthermore, our single defect interaction estimates are in fact an upper bound on this quantity.

In conclusion, we have proposed a mechanism which may explain why local defects, which must certainly be present on even the smoothest surfaces, might not prevent a stiff monolayer film from sliding under the ex- tremely weak inertial forces that occur in a quartz microbalance experiment, if the defect potential has a range greater than about 1.5 film lattice spacings. Our proposed mechanism is supported by a simple model calculation, based on the fact that each defect can interact with several film atoms at a time. As a consequence, the resultant forces on the film are much less than the maximum force provided by the defect potential. Lattice vibrations are able to provide an oscillating force which is larger than the net static force due to the defect potential acting on the film. This can lead to a high degree of thermal activation of the sliding of the film.

\section{Acknowledgments}

J.B. Sokoloff wishes to thank the Department of Energy (Grant DE-FG02-96ER45585).
[1] J. Krim, D. H. Solina and R. Chiarello, Phys. Rev. Lett. 66, 181 (1991); C. Daly and J. Krim, Phys. Rev. Lett. 76,803 (1996).

[2] J. B. Sokoloff, Phys. Rev. B 42, 760,6745(E) (1990); Phys. Rev. B47,6106 (1993); Wear 167, 59 (1993); Phys. Rev. Lett. 71, 3450 (1993); J. B. Sokoloff, Phys. Rev. B 51, 15573 (1995); J. B. Sokoloff and M. S. Tomassone, Phys. Rev. B 57, 4888-4897 (1998); B. N. J. Persson and A. I. Volokitin, J. Phys.: Condensed Matter 9, 2869 (1997).

[3] M. S. Tomassone and J. B. Sokoloff, Phys. Rev. B 60, 4005 (1999).

[4] R. L. Renner, J. E. Rutledge and P. Taborek, Phys Rev Lett 83, 1261 (1999).

[5] A. Dayo, W. Alnasrallah and J. Krim, Phys Rev. Lett. 80, 1690 (1998).
[6] B. L. Mason, S. M. Winder and J. Krim, Tribology Letters 10, 59 (2001).

[7] A. I. Larkin and Yu. N. Ovchinikov, J. Low Temp. Phys. 34, 409 (1979).

[8] N. W. Ashcroft and N. D. Mermin, "Solid State Physics," Holt Rinehart and Winston, Philadelphia, 1976), p. 137.

[9] E. Jahnke and F. Emde, "Tables of Functions," 4 th edition (Dover Publications, New York, 1938.

[10] Landau L. D. and Lifshits E. M., Theory of Elasticity, 2nd edition (Pergamon Press, New York, 1975).

[11] H. Fukuyama and P. A. Lee, Phys. Rev. B17, 535 (1977); P. A. Lee and T. M. Rice, Phys. Rev. B19 (1979) 3970; D. S. Fisher, Phys. Rev. B 31, 1396 (1985); J. B. Sokoloff, Phys. Rev. Lett. 86, 3312 (2001); Phys. Rev. B 65, 115415 (2002); J. B. Sokoloff and I. Webman, unpublished. 